\title{
Seroconversion of Indirect Immunofluorescent Antibody IgM and IgG in Melioidosis
}

\author{
Arumugam Janaki ${ }^{1}$, How Soon $\mathrm{Hin}^{2}$, Ummu Afeera Zainulabid ${ }^{3}$, Mohammed Imad A. Mustafa \\ Mahmud $^{4}$, Jamaluddin Ab. Rahman ${ }^{5}$, Ahmad Kashfi Ab. Rahman ${ }^{6}$, Yeh Chunn Kuan ${ }^{7}$
}

\begin{abstract}
${ }^{1}$ Science Officer, Department of Basic Medical Science, Hospital Tengku Ampuan Afzan, Kuantan, Pahang, Malaysia; ${ }^{2}$ Pulmonologist and Medical Lecturer, Department of Internal Medicine, Kulliyyah of Medicine, International Islamic University Malaysia, Kuantan, Pahang, Malaysia; ${ }^{3}$ Internal Medicine Specialist and Medical Lecturer, Department of Internal Medicine, Kulliyyah of Medicine, International Islamic University Malaysia, Kuantan, Pahang, Malaysia; ${ }^{4}$ Medical Lecturer, Department of Basic Medical Science, Kulliyyah of Medicine, International Islamic University Malaysia, Kuantan, Pahang, Malaysia; ${ }^{5}$ Public Health Consultant, Department of Community Medicine, Kulliyyah of Medicine, International Islamic University Malaysia, Kuantan, Pahang, Malaysia; ${ }^{6}$ Infectious Disease Consultant, Department of Internal Medicine, Hospital Sultanah Nur Zahirah, Terengganu, Malaysia; ${ }^{7}$ Pulmonologist and Ex Medical Lecturer, Department of Internal Medicine, Kulliyyah of Medicine, International Islamic University Malaysia, Kuantan, Pahang, Malaysia
\end{abstract}

[Received: 12 October 2020; Accepted: 30 November 2020; Published: 1 December 2020]

\begin{abstract}
Background: There are several serological tests used in the diagnosis of melioidosis. However, the interpretation of the results can be problematic in endemic areas because there might be a high background positivity due to previous exposure. Objective: This study aimed to determine the usefulness of Indirect Immunofluorescent Antibody (IFA) IgM and IgG in the diagnosis of melioidosis and the time for seroconversion. Methodology: We prospectively studied the trend of IFA IgM and IgG in 40 patients with culture-confirmed melioidosis over three months at six different time points (days 1, 8, 15, 30, 60, and 90). Results: From the results, $37.5 \%$ and $32.5 \%$ of patients had IFA IgM and IgG of $\leq 1: 20$ respectively on day 1 when the blood culture was positive. The natural $\log (\ln )$ of the titres was used for the analysis. Repeated measures ANOVA showed significant changes in both $\operatorname{IgM}(\mathrm{p}=0.001)$ and $\operatorname{IgG}(\mathrm{p}=0.045)$ respectively throughout six sampling time points. Both the means of $\ln \operatorname{IgM}$ and $\ln \operatorname{IgG}$ peaked at day 15 . At day 90 , mean $\ln$ IgG remained high but mean $\ln$ IgM dropped to a level below that of day one. All culture-confirmed melioidosis patients recorded a maximum IFA IgM titre of at least 1: 80, but all subsequently dropped to below this level at day 90. Conclusion: IFA IgM is a more useful diagnostic serological marker than IFA IgG in acute melioidosis. [Bangladesh Journal of Infectious Diseases, December 2020;7(2):61-66]
\end{abstract}

Keywords: melioidosis, serology, IFA IgM, IFA IgG, seroconversion

Correspondence: Ummu Afeera Zainulabid, Assistant Professor, Kulliyyah of Medicine, International Islamic University Malaysia, Jalan Sultan Ahmad Shah, 25200 Kuantan, Pahang, Malaysia; Email: ummuafeera@iium.edu.my; Cell no.: 09-5704712 / 09-5912500

Conflict of interest: No conflict of interest

Funding agency: This study was funded by an IIUM internal fund.

Contribution to authors: AJ, HSH, MIAMM, JAR, AKAR, YCK involved in protocol preparation, data collection and literature search up to manuscript writing. AJ, HSH, MIAMM, JAR, UAZ involved in literature search, preparation and revision of this manuscript.

How to cite this article: Janaki A, How SH, Ummu Afeera Z, Mohammed Imad AMM, Jamalludin AR, Ahmad Kashfi AR, Kuan YC. Seroconversion of Indirect Immunofluorescent Antibody (IFA) IgM and IgG in Melioidosis. Bangladesh J Infect Dis 2020;7(2):61-66

Copyright: (02020. Janaki et al. Published by Bangladesh Journal of Infectious Diseases. This article is published under the Creative Commons CC BY-NC License (https://creativecommons.org/licenses/by-nc/4.0/). This license permits use, distribution and reproduction in any medium, provided the original work is properly cited, and is not used for commercial purposes. 


\section{Introduction}

Melioidosis is caused by a gram-negative bacillus, Burkholderia pseudomallei, a saprophyte commonly found in the soil and freshwater in tropical and subtropical regions. The disease is endemic in Southeast Asia and tropical Australia ${ }^{1-6}$. In recent years, melioidosis is increasingly reported from other regions such as India, China, and the islands in the Pacific and Indian Oceans ${ }^{7-9}$. The spread of melioidosis to non-tropical countries could be attributed to returning travellers from endemic $\operatorname{areas}^{10-11}$. Despite the newly developed treatment modalities for melioidosis, its overall mortality rate remains very high $^{2-4}$.

To reduce mortality, it is crucial to diagnose melioidosis in the early stage. Currently, the gold standard for definitive diagnosis of melioidosis is the conventional culture method. However, there are some issues with this method. Firstly, the sensitivity of the culture method may be low in patients who have been taking antimicrobial treatment. This is due to the low bacterial concentration in clinical specimens, especially blood samples of these patients. Furthermore, some countries do not use selective medium in the culture. As a result, the culturing of samples from non-sterile sites using a non-selective media may lead to an overgrowth of normal flora, thus inhibiting the growth of the pathogen. Moreover, it takes 3 to 5 days to confirm the diagnosis via the culture method, thus resulting in the delay of the initiation of appropriate treatment 12. Therefore, more rapid and sensitive diagnostic methods are required to reduce the mortality rate of melioidosis.

Another diagnostic method for melioidosis, the Indirect Hemagglutination Assay (IHA) has been used for a long time in many countries to detect the total antibodies. However, this assay has low sensitivity and specificity, especially in endemic countries. A study in Thailand showed that IHA titre of $\geq 1: 40$ was found in $47.1 \%$ of blood donors and $29.7 \%$ of sera submitted for other tests ${ }^{13}$. Some donors even recorded positive titres of 1:1280. Indirect Immunofluorescent Antibody (IFA), another test that was used in the same study, showed better sensitivity and specificity than IHA. Jamuna et al. showed that the sensitivity and specificity of IFA total antibodies were more than $90 \%$ in the diagnosis of melioidosis ${ }^{14}$.

Although the detection of antibody is useful in the diagnosis and treatment of patients with melioidosis, the existence of background seropositivity in healthy individuals in endemic areas and the delay in seroconversion in some patients make this approach unreliable ${ }^{15}$. To date, there has been no study that assesses the changes in the level of antibodies over time in patients and the time needed for seroconversion. There is also a lack of evidence demonstrating whether IgM or IgG would be a better diagnostic test. Thus, this study was aimed to prospectively study the trend of IFA IgM and IgG in patients with culture-confirmed melioidosis to determine which a better diagnostic test is and to determine the time of seroconversion.

\section{Methodology}

This prospective observational study recruited patients with culture-confirmed melioidosis from two major hospitals on the east coast of peninsular Malaysia, namely Hospital Tengku Ampuan Afzan (HTAA), Kuantan, Pahang and Hospital Sultanah Nur Zahirah (HSNZ), Kuala Terengganu, Terengganu from January 2014 to December 2015. The diagnosis of melioidosis was confirmed by the conventional culture method. The bacterial culture and identification were performed at the microbiology laboratories in both hospitals. This study was approved by the IIUM Research Ethics Committee (IREC) (Registration no: IREC 303) and the Medical Research \& Ethics Committee (MREC), Ministry of Health Malaysia (NMRR-14-114121985). Informed consent was obtained from all patients. For those who were confused or unable to communicate due to medical infirmity, informed consent was obtained from the next of kin or guardian. Baseline demographic and clinical data were recorded. During the study period, melioidosis patients were managed by physicians from the respective hospitals. Blood samples were taken over three months on six different days (day 1, 8, 15, 30, 60, and 90) for IFA $\operatorname{IgM}$ and IgG. A total of 80 control subjects were recruited, consisting of 40 patients without febrile illness who presented to the hospitals for blood tests due to hypertension and diabetes mellitus and another 40 patients with other bacterial infections that were culture-confirmed. Only a single blood sample was taken from each of the control subjects.

Bacterial Strains: The strain of B. pseudomallei used for IFA was obtained from the blood culture of a patient with clinical manifestation of melioidosis at HTAA. Blood cultures for all suspected melioidosis patients were done using the BACTEC blood culture system. The positive blood bottle was then subcultured in blood agar, MacConkey agar, and Francis agar. Culture plates were incubated at 35-37 ${ }^{\circ} \mathrm{C}$ for 24 hours. After incubation, colonies were identified based on their morphological features, i.e. 
the presence of yellow haze on Francis media. They were also confirmed using a commercial identification kit, API 20NE System (Biomeriux, France). A bacterial suspension in Trypticase Soy Broth (TSB) was prepared from pure colonies that were heat-inactivated before further processing.

Indirect Immunofluorescent Antibody Testing (IFA): The IFA was carried out as described by Ashdown ${ }^{16}$ with some modifications. The bacterial antigen was washed 3 times in Phosphate Buffer Solution (PBS) pH 7.3 and then resuspended in PBS $\mathrm{pH} 8.5$ and stored at $4-8{ }^{\circ} \mathrm{C}$ as a stock antigen for slide preparation. At this temperature, the stock antigen can be stored up to 3 weeks. The working antigen was then prepared by diluting stock antigen in PBS ( $\mathrm{pH}$ 8.5) to achieve turbidity of 0.5 McFarland standard. The working antigen was used to coat the 12 wells of the Teflon-coated slides before being air-dried and fixed with cold acetone. Sera of the patients were serially diluted two-fold in PBS (pH 7.3), starting from a 1:10 dilution to 1:160. Each dilution was then overlaid onto the antigen wells and incubated at $37^{\circ} \mathrm{C}$ for 30 minutes in a moist chamber. A fluoresce in isothiocynate (FITC) tagged antihuman globulin, specific for IgM and IgG (Kirkegaard \& Perry Laboratories, United Kingdom), was separately added after washing the slides with PBS ( $\mathrm{pH}$ 7.3) 3 times. Then, the slides were again incubated for a further 30 minutes in a moist chamber at $37^{\circ} \mathrm{C}$ before being washed with PBS (pH 7.3) and mounted with buffered glycerol. Positive and negative control sera were included in each batch of tests. Finally, the stained slides were examined under a fluorescent microscope at 40x magnification. A positive result was defined by the appearance of apple-green fluorescence of the bacilli of $B$. pseudomallei. If high positive cell counts were noted at a 1:160 dilution, the test was repeated at higher dilutions of 1:320, 1:640, and 1:1280. If a negative result was noted at 1:10 dilution, the result was recorded as $<1: 10$.

Statistical Methods: The demographic data of patients with melioidosis and control group subjects were compared using one-way ANOVA for the mean age and Chi-square for the proportion of gender and diabetic status. Due to the exponential nature of $\operatorname{IgM}$ and $\operatorname{IgG}$ titre measurement, the means of antibody titres in each immunoglobulin class were 1n-transformed. All titres of 1: $<10$ were presumed as equal to 5 to avoid any missing values during statistical analysis. Repeated measures ANOVA testing was performed on the changes in the means of IgM and IgG throughout the six sampling times. Bonferroni post hoc test was done when required.

\section{Results}

A total of 40 patients who had cultured-confirmed melioidosis were recruited for this study. However, only nine $(22.5 \%)$ of the patients were able to provide the blood samples for all the six time points from day 1 until day 90 . The majority of recruited patients passed away (52.5\%) due to the infection, leading to incomplete blood sample collection. One sample was collected in 14 patients, two samples were collected in three patients, three and four samples were collected in two of the patients respectively. Another ten patients defaulted their follow up despite multiple reminders (one sample was collected in two patients, two samples were collected in five patients, four samples were collected in one patient, and five samples were collected in two patients). Two patients missed their appointments on day 30 but came for subsequent follow-up at day 60 and day 90. Overall, 112 sets of sera and plasma were collected throughout this study (Table 1).

\section{Table 1: Summary of the number of patients with melioidosis at each stage of sampling}

\begin{tabular}{|l|c|c|c|c|c|c|c|}
\hline $\begin{array}{l}\text { Status of } \\
\text { Patients } \\
\text { after } \\
\text { Sampling }\end{array}$ & \multicolumn{6}{|c|}{ Day of sampling } & \multicolumn{2}{c|}{ Total } \\
\cline { 2 - 7 } & - & $\infty$ & 2 & 8 & 8 & 8 & \\
\hline $\begin{array}{l}\text { Total } \\
(\mathrm{n}=40)\end{array}$ & 40 & 24 & 16 & 12 & 11 & 9 & $\begin{array}{c}112 / 240 \\
(46.7 \%)\end{array}$ \\
\hline Deceased & 14 & 3 & 2 & 2 & 0 & 0 & $\begin{array}{c}21 / 40 \\
(52.5 \%)\end{array}$ \\
\hline Defaulted & 2 & 5 & $2 *$ & 1 & 2 & 0 & $\begin{array}{c}10 / 40 \\
(25 \%)\end{array}$ \\
\hline Remained & 40 & 24 & 16 & 12 & 11 & 9 & $\begin{array}{c}9 / 40 \\
(22.5 \%)\end{array}$ \\
\hline
\end{tabular}

* = patients missed the appointment on day 30 ( \pm 2 days)

The mean $\ln \operatorname{IgM}$ and $\mathrm{IgG}$ in the melioidosis group was significantly higher compared to the control group with or without other infections (Figure I). The optimal cut-off points for IFA IgM and IFA IgG were determined as 1:20 and 1:80 respectively (detailed analysis described elsewhere). Only 11 patients $(27.5 \%)$ had IFA IgM of $<1: 20$ on day 1 . Four of them had IFA IgG of 1:80 or more. Of these 11 patients, five died within a week, thus repeat serology could not be done. Six patients had serology titres of at least 1:40 on day 8. Fourteen patients $(35 \%)$ had IFA IgG of 1:40 or less on day 1. Six patients had repeated IFA IgG on day 8 when the titres increased to at least 1:80. Only nine subjects completed all the six time points. Repeated measures 
ANOVA for data obtained from these nine patients showed significant changes of both specific IgM and specific IgG antibodies over the test period (partial eta $=0.598, \mathrm{p}<0.001$ and partial eta $=0.410, \mathrm{p}=0.001$ respectively).

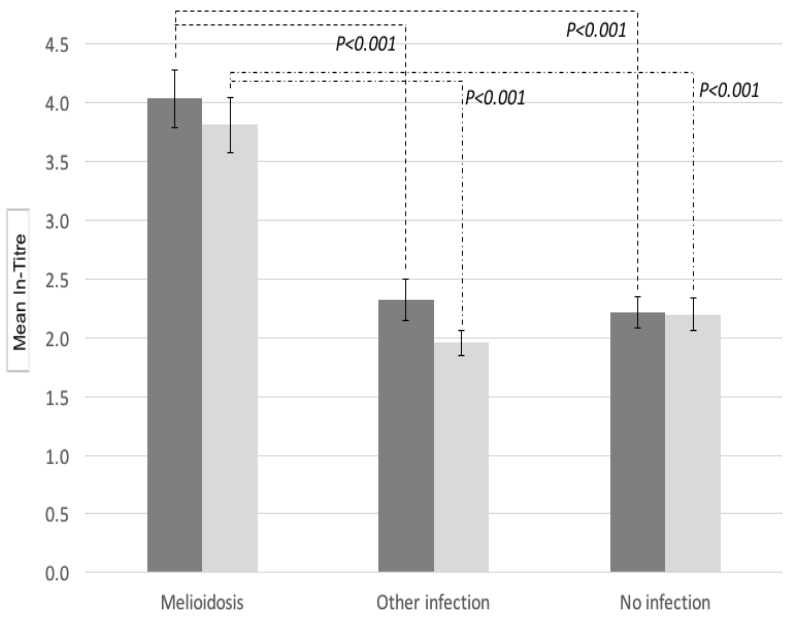

Figure I: Mean In IgM (dark grey bar) and In IgG (light grey bar) Titres at day 1 by the status of infection

Figure II shows the serological responses of specific IgM and IgG antibodies in patients with melioidosis over the study period. Both mean $\ln$ IgM and $\ln \mathrm{IgG}$ rose and peaked at day 15 . At day 90 , the mean $\ln$ IgG remained high but the mean $\ln$ IgM dropped to a level below that of day 1 .

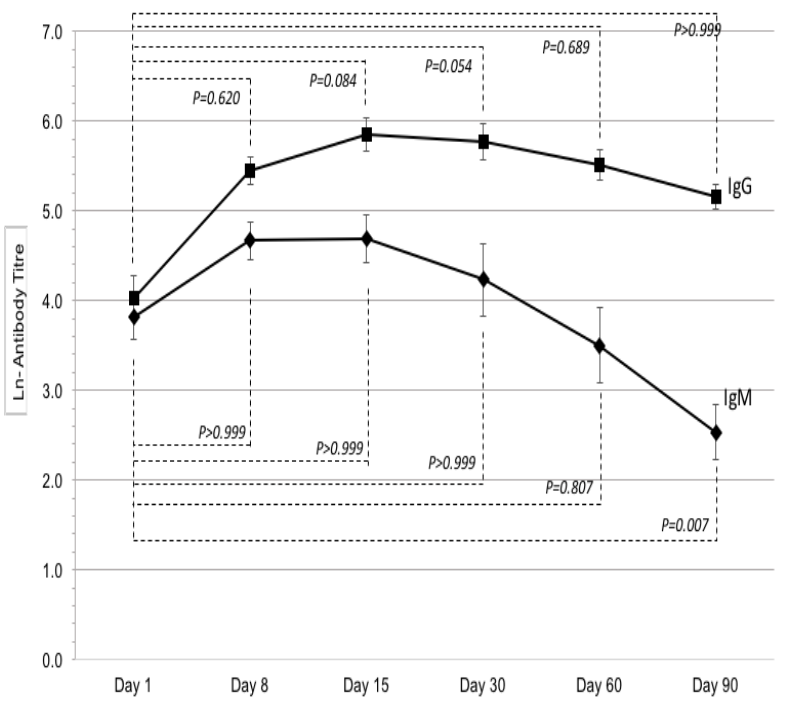

Figure II: Mean In IgM and In IgG titres and 95\% Confidence Interval of mean ln over time

Figure III shows the serological titres of $\operatorname{IgM}$ and IgG over the study period. All culture-confirmed melioidosis patients had a maximum IFA IgM titre of at least 1: 80, all of which dropped subsequently. Among the nine patients who had IFA IgM done at day 90, 4 had titres of 1:5, 3 had antibody titres that declined to 1:20, and another 2 declined to 1:40. Nine patients had $\operatorname{IgG}$ repeated at day 90 when all titres were $1: 80$ or more.

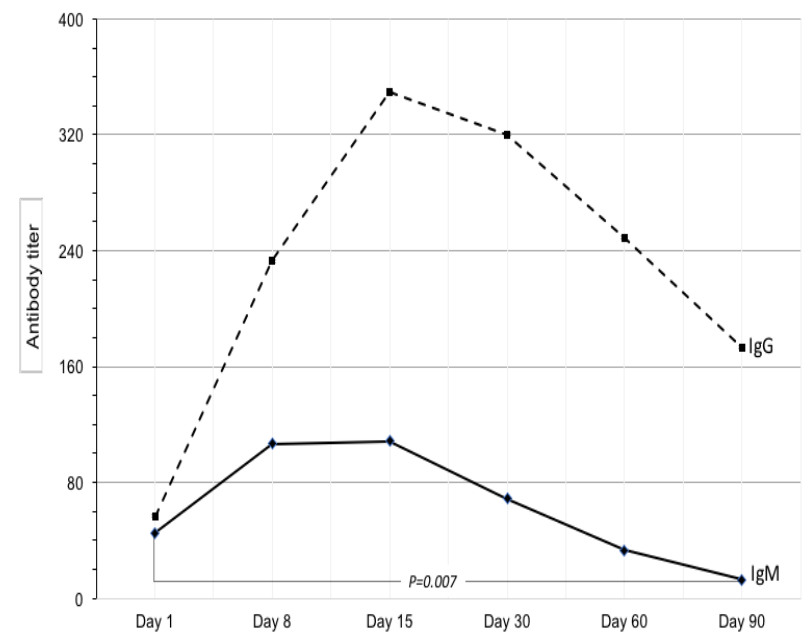

Figure III: Mean IgM and IgG titres over time

\section{Discussion}

A study in North-Eastern Thailand showed the sensitivity of the IHA test to be $95 \%$ but specificity to be only $59 \%$ when the cut-off titre was $1: 20^{7}$. This could be attributed to a high background positive serology. In Australia, IHA of 1:40 is used as the cutoff level. Harris et a ${ }^{17}$ retrospectively reviewed 140 culture-confirmed melioidosis patients and found that $51 \%$ of them had IHA of $\geq 1: 40$. It is well known in the field of serology that the use of a higher cutoff level will reduce the sensitivity but improve the specificity of the test. However, surprisingly, the same study found that in nearly half of the patients who had IHA repeated after 1 to 55 months, $26 \%$ of them had persistently negative test results, $15 \%$ showed seroconversion and $17 \%$ experienced seroreversion. One of the possible reasons could be that seroconversion was missed because the test was not repeated at frequent intervals. It could also be due to the poor sensitivity of the IHA test. In this study, all patients with negative IFA IgM showed seroconversion on day 8 and the mean $\ln$ of $\operatorname{IgM}$ antibody titres peaked at day 15 . Based on this, repeated serology should be done between day 8 to day 15 to detect or confirm seroconversion.

The IFA IgM test, described by Ashdown et $\mathrm{al}^{18}$, showed better sensitivity and specificity. This test was also performed in Thailand using the same method and a cut-off level of $\geq 1: 80$ to indicate positive cultures. In this study, a high correlation of 
IFA IgM seropositivity was observed in bacteriologically proven cases, whereby IFA IgM was positive in six out of seven patients with acute sera. Based on this study that included 40 patients with acute sera, a titre of 1:20 as the cut-off level would produce sensitivity and specificity of $72.5 \%$ and $80.0 \%$, with positive and negative predictive values of $64.4 \%$ and $85.3 \%$ respectively. However, if the titre of $\geq 1: 80$ was used as the cut-off level, the sensitivity dropped to $60 \%$, which was much lower than the Thailand study. However, all of the patients with IFA IgM titres < 1:80 subsequently showed an increase in titre level to $1: 80$ or more. This indicated that an IFA IgM of $\geq 1: 80$ is highly suggestive of melioidosis. Nevertheless, in control group, $6.25 \%$ of patients also had IFA IgM of 1:80. These could be false-positive cases as most control subjects were made up of patients without infection or with infections caused by bacteria other than $B$. pseudomallei. The falsely high titre could be an anamnestic response to previous melioidosis infection. A similar finding was reported in a Thai study in which four of their patients showed persistently raised IgM titre up to 3 years ${ }^{18}$. Another reason might be the incomplete clearance or killing of viable microorganisms in the tissues ${ }^{19}$. This could explain why IFA IgM was positive in patients without evidence of infection. In the same study, IFA IgM was positive in 1 of 227 blood donors ${ }^{18}$. Therefore, it is a common consensus that positive IFA IgM without evidence of infection does not warrant any treatment. However, it remains unknown whether attending physicians should follow up and monitor the IgM levels in melioidosis patients to ascertain the risk of reactivation.

In the course of any microbial infections, it has been established that IgM plays a vital role by providing specific high avidity (but low affinity) first-line armour prior to the production of IgG. The level of IgM increases rapidly and becomes detectable after any pathogenic infection, reaching a maximum level within 10 to 14 days post-infection. After that, the IgM level will decline and becomes undetectable after 3 to 6 months once the infection is cleared ${ }^{19}$. This study, however, showed that IFA IgM peaked on day 8 . This could be explained by the fact that day 1 in the study was based on the date of blood sampling rather than the day of infection, thus the reason why our IFA IgM peaked at day 8 rather than later. Furthermore, IFA IgM showed more dynamic changes during acute infection in this study and declined faster than IgG during the three months of follow-up, hence suggesting that IFA IgM is a better early indicator of acute infection. By comparison, IgG reached a much higher peak level and maintained a relatively high level until day 90 . Thus, relying on IFA IgG as the sole diagnostic serological test may lead to a high false positive rate.

Currently multiple antigens ELISA test ${ }^{20}$ on TssD-5, Omp3, smBpF4, and Omp85 have been established for the diagnosis of melioidosis. Their sensitivity and specificity were reported as $88.2 \%$ and $96 \%$, thus they may be a better test compared to IFA IgM. For example, TssD-5 alone had a sensitivity and specificity of $96 \%$ and $71 \%$. Another study showed that the indirect ELISA using Omp $\mathrm{A}$ as an antigen achieved $95 \%$ sensitivity and $98 \%$ specificity ${ }^{21}$. Clearly, the tests with higher specificity will be useful in endemic countries as they will reduce the false positive result. However, these tests are still in their infancy stages and they should be validated in larger clinical trials, preferably in the endemic countries before their wider application.

\section{Conclusion}

In conclusion, IFA $\operatorname{IgM}$ is more useful in the diagnosis of acute melioidosis than IgG. This test should be repeated on day 8 or day 15 to look for seroconversion in cases with low initial titres.

Acknowledgement: We thank all the microbiology staff at Hospital Tengku Ampuan Afzan (HTAA), Kuantan, Kulliyyah of Medicine, IIUM and Hospital Sultanah Nur Zahirah (HSNZ), Kuala Terengganu for their assistance and provision of serum samples.

\section{References}

1. Currie BJ, Fisher DA, Howard DM, et al. Endemic melioidosis in tropical northern Australia: a 10-year prospective study and review of the literature. Clin Infect Dis. 2000;31(4):981-986.

2. Puthucheary SD, Parasakthi N, Lee MK. Septicaemic melioidosis: a review of 50 cases from Malaysia. Trans R Soc Trop Med Hyg. 1992;86(6):683-685.

3. How $\mathrm{SH}, \mathrm{Ng} \mathrm{KH}$, Jamalludin AR, Shah A, Rathor Y. Melioidosis in Pahang, Malaysia. Med J Malaysia. 2005;60(5):606-613.

4. Suputtamongkol Y, Hall AJ, Dance DA, et al. The epidemiology of melioidosis in Ubon Ratchatani, northeast Thailand. Int J Epidemiol. 1994;23(5):1082-1090.

5. Heng BH, Goh KT, Yap EH, Loh $\mathrm{H}$, Yeo $\mathrm{M}$. Epidemiological surveillance of melioidosis in Singapore. Ann Acad Med Singapore. 1998;27(4):478-484

6. Chaowagul W, White NJ, Dance DA, Wattanagoon Y, Naigowit P, Davis TM, Looareesuwan S, Pitakwatchara N. Melioidosis: a major cause of community-acquired septicemia in northeastern Thailand. Journal of Infectious Diseases. 1989;159(5):890-9

7. Mukhopadhyay A, Lee KH, Tambyah PA. Bacteraemic melioidosis pneumonia: impact on outcome, clinical and radiological features. J Infect. 2004;48(4):334-338.

8. Dance DA. Melioidosis: the tip of the iceberg? Clin Microbiol Rev. 1991;4(1):52-60.

9. Inglis TJ, Rolim DB, Sousa Ade Q. Melioidosis in the Americas. Am J Trop Med Hyg. 2006;75(5):947-954. 
10. Currie BJ. Melioidosis: an important cause of pneumonia in residents of and travellers returned from endemic regions. Eur Respir J. 2003;22(3):542-550.

11. Carlson P, Seppänen M. Melioidosis presenting as urinary tract infection in a previously healthy tourist. Scand J Infect Dis. 2000;32(1):92-93.

12. Limmathurotsakul D, Jamsen K, Arayawichanont A, Simpson JA, White LJ, et al. Defining the True Sensitivity of Culture for the Diagnosis of Melioidosis Using Bayesian Latent Class Models. PLOS ONE 2010;5(8): e12485.

13. Khupulsup K, Petchclai B. Application of indirect hemagglutination test and indirect fluorescent antibody test for IgM antibody for diagnosis of melioidosis in Thailand. Am J Trop Med Hyg. 1986;35(2):366-369.

14. Vadivelu J, Puthucheary SD. Diagnostic and prognostic value of an immunofluorescent assay for melioidosis. Am J Trop Med Hyg. 2000;62(2):297-300.

15. Wuthiekanun V, Chierakul W, Langa S, et al. Development of antibodies to Burkholderia pseudomallei during childhood in melioidosis-endemic northeast Thailand. Am J Trop Med Hyg. 2006;74(6):1074-1075.
16. Ashdown LR. Demonstration of human antibodies to Pseudomonas pseudomallei by indirect fluorescent antibody staining. Pathology. 1981;13(3):597-601.

17. Harris PN, Ketheesan N, Owens L, Norton RE. Clinical features that affect indirect-hemagglutination-assay responses to Burkholderia pseudomallei. Clin Vaccine Immunol. 2009;16(6):924-930

18. Ashdown LR. Relationship and significance of specific immunoglobulin $\mathrm{M}$ antibody response in clinical and subclinical melioidosis. J Clin Microbiol. 1981;14(4):361-364.

19. Vasu C, Vadivelu J, Puthucheary SD. The humoral immune response in melioidosis patients during therapy. Infection. 2003;31(1):24-30.

20. Hara Y, Chin CY, Mohamed R, Puthucheary SD, Nathan S. Multiple-antigen ELISA for melioidosis--a novel approach to the improved serodiagnosis of melioidosis. BMC Infect Dis. 2013;13:165

21. Allwood EM, Logue CA, Hafner GJ, et al. Evaluation of recombinant antigens for diagnosis of melioidosis. FEMS Immunol Med Microbiol. 2008;54(1):144-153 